\title{
PN ABUNDANCES IN DIFFERENT GALACTIC SYSTEMS
}

\author{
R.E.S. CLEGG \\ Royal Greenwich Observatory, Madingley Road, Cambridge CB3 OEZ, U.K.
}

\begin{abstract}
Developments in the measurement of abundances in planetary nebulae since IAU Symposium 131 are reviewed. Present uncertainties and outstanding problems in the interpretation of emission-line measurements are discussed. Results for different samples of PN are reviewed, in terms of the galactic site. Results for the Galactic disk, Galactic Bulge, Galactic Halo, LMC \& SMC, Fornax Dwarf galaxy and M31 are discussed. Attention is drawn to the importance of Magellanic Cloud samples for testing dredge-up theory, and to the strong variation of PN C/O ratios with the local site's metallicity and age. New results for carbon in Cloud PNs are presented. Important problems in the interpretation of the metallicity of Bulge PNs are highlighted. Attention is drawn to the idea that metal-rich stellar populations do not produce PNs, such stars leaving the lower AGB early.
\end{abstract}

\section{Introduction \& Overview}

In this review I will concentrate on developments in the field of PN abundances since the last IAU Symposium (No 131, in Mexico City) was held. In section 1 I will cover the general abundance pattern, recent surveys and compilations, and some current uncertainties in deriving abundances. In Section 2 I discuss briefly results for the nearby Galactic disk. Sections 3, 4, 5 \& 6 cover the Halo, Bulge, Magellanic Clouds (hereafter, 'Clouds') and other extragalactic systems, respectively, with new results for Cloud PNs being presented in Sec. 5.

Some recent reviews on abundances and related properties were given by Clegg (1989, 1991), Peimbert $(1990,1992)$ and Henry (1990). The systematics of a large set of Galactic PN abundances were studied by Perinotto (1992).

Three interesting examples of how PN properties vary (or not!) as a function of 'metallicity' or galactic site are given here. Note that different metallicity regions usually have different chemical evolution histories and ages (and hence different $A G B$ star masses).

Firstly, Henry (1989) compiled $\mathrm{O} / \mathrm{H}$ and $\mathrm{Ne} / \mathrm{H}$ ratios for $\mathrm{PNs}$ in the disk, Bulge, Halo and Clouds, i.e. over a large spread in metallicity. The Ne/O ratio is remarkably constant at 0.17 by number, and Henry showed that this puts a significant constraint on the constancy of the ratio of the numbers of massive stars above $15 \mathrm{M}_{\odot}$ (since there the relative yields of $\mathrm{O}$ and $\mathrm{Ne}$ vary with mass).

A second example is provided by the idea that metal-rich stellar populations may not produce many PNs. This was suggested to explain the UV-excess seen in elliptical galaxies (Greggio \& Renzini 1989, Brocato et al. 1990) and justified by later stellar evolutionary models (Castellani \& Tornambe 1991, 1992). The suggestion is that, in old systems of $\sim 2 \times$ Solar metallicity, high mass-loss results in stars only reaching the early AGB (non-thermally-pulsing) before the envelope mass tends to zero, and the stars move to the left in the HR diagram without ejection of a PN. A population of hot 'post-early-AGB' stars rather than PN central stars would contribute to the far-UV light in the most metal-rich galaxy cores, such as NGC1399 (Castellani \& Tornambe 1990, Ferguson \& Davidson 1993). Ferguson \& Davidsen show that the number of PNs per unit bolometric luminosity in early-type galaxies 
decreases as the $(1550 \AA-\mathrm{V})$ colour get bluer, a trend which supports the above hypothesis. Peimbert (1990) noticed that the PN number and the PN birthrate per unit luminosity correlated with galaxian $\mathrm{B}-\mathrm{V}$ colour and with $\mathrm{M}_{B O L}$, which may be closely related effects.

If correct, this idea would affect the interpretation of mean PN abundances in an old, metal-rich (Solar or above) galactic site: the mean planetary nebula $\mathrm{O} / \mathrm{H}$ value would be lower than the mean stellar value, for example.

Lastly, the variation of nebular $\mathrm{C} / \mathrm{O}$ ratio with galactic site is particularly strong. The percentage of $\mathrm{PNs}$ having $\mathrm{C} / \mathrm{O}>1$ varies from 18 (Bulge); 50 ('Southern' PNs, Kingsburgh \& Barlow these proceedings); 65 (local disk, Zuckerman \& Aller 1987); 60 (Halo objects) and almost 100 (Magellanic Clouds; see below). Although this is partly due to a 'titration effect' (less $\mathrm{C}$ needed when the $\mathrm{O} / \mathrm{H}$ ratio is low in a stellar envelope) it is also due to the 'efficiency' of the 3rd dredge-up (which can include opacity, mixing, and envelope-mass effects).

\section{Abundance Determinations}

The main current uncertainties arise from uncertainties in:

- Depletion of atoms in grains (especially, for O and C). However, recent determinations of PN dust/gas ratios by mass (reviewed in article by Barlow) are so low $\left(\sim 10^{-4}\right)$ that the required depletions of $\mathrm{C} \& \mathrm{O}$ are below $20 \%$.

- Ionization correction factors for sulphur \& argon.

- Temperature fluctuations in nebulae - is the fluctuation parameter $t^{2}$ significant? A fresh stimulus for this is the finding from new CCD data that measured Balmer continuum temperatures are lower than [O III] values (Liu \& Danziger 1993). The difference corresponds to a parameter $t^{2}=0.035$.

- Whether localized shock-excitation is common or significant. Peimbert et al. (1991) described situations in which PNs \& H II regions might have shock-excitation. Middlemass et al. (1991) presented a shock model for PN giant halos; it was found that derived $\mathrm{O} / \mathrm{H} \& \mathrm{Ne} / \mathrm{H}$ ratios could be a factor 2 too low. But, they also showed that such an effect would not operate in the main, bright part of the nebulae. Several Type I PNs in the SMC (discussed below) have $\mathrm{T}_{e}\left(\mathrm{O}^{++}\right) \sim 25,000 \mathrm{~K}$, which cannot arise from photo-ionization but could be due to shock-excitation.

- The still un-resolved discrepancy between $\mathrm{C}^{++} / \mathrm{H}^{+}$ratios derived from the [C III] 1908 and C II $4267 \AA$ lines. From IUE \& optical spectra of Cloud PNs, Walton et al. (1991) found that $\mathrm{T}_{e}\left(\mathrm{C}^{++}\right)$is much less than $\mathrm{T}_{e}\left(\mathrm{O}^{++}\right)$, disagreeing with simple expectations and with photo-ionization models. The problem is not confined to Galactic PNs! The $\mathrm{C}^{++} / \mathrm{H}^{+}$ratios would be significantly increased for the Cloud PNs if the $\mathrm{T}_{e}\left(\mathrm{C}^{++}\right)$values were used to interpret the C III]1908 $\AA$ fluxes. TorresPeimbert et al. (1990) made a photo-ionization model of NGC 4361 with a gradient in the $\mathrm{C} / \mathrm{H}$ ratio, and obtained good fits to $\mathrm{C}$ line strengths. This provides a possible answer to this old problem.

From analysis of near-IR HF lines, Jorissen et al. (1992) found fluorine to be over-abundant in many red giants, with $\mathrm{F} / \mathrm{H}$ up to $30 \times$ Solar in carbon stars. The $\mathrm{F} / \mathrm{O} \& \mathrm{C} / \mathrm{O}$ ratios were well-correlated. The suggested mechanism for ${ }^{19} \mathrm{~F}$ production was $\alpha$-particle capture on ${ }^{14} \mathrm{~N}$ in the He-burning shell at the AGB 
stage. Aller \& Czyzak (1983) do quote a mean PN log F abundance of 4.6 (the same as the Solar value adopted by Jorissen et al.), but presumably this is based on guessed atomic data. It would be useful to improve the accuracy of $\mathrm{PN} \mathrm{F/H}$ ratios. The strongest (!) line seen in PNs is [F IV]4059 (analogue of [O III]5007); new deep CCD spectra are required, together with atomic data for the FIV lower levels. Even rough abundances accurate to a factor 2 would be of value, in view of the large reported enhancement factors in red giants.

\section{The Galactic Disk}

Fresh abundance sets have been given by Aller \& Keyes (1987), Henry (1989), de Freitas Pacheco et al. (1991, 1992), Koeppen et al. (1991) \& Kingsburgh \& Barlow (Poster VI-225) - the last three studies concentrate on Southern PNs which may be systematically closer to the Galactic Centre than other samples of bright nebulae. A large compilation of Galactic PN abundances was given by Perinotto (1992). Abundances in large PNs were studied by Kaler et al. (1990), and abundance gradients were studied by Faundez-Abans \& Maciel (1988) \& Maciel (1991) (see also Posters VI-232 \& 233).

The situation of 1987 still stands: the average PN has abundances of He, C \& $\mathrm{N}$ (only) enhanced over the values for H II regions in the same galactic site. The increases are attributed to the 1st, 2nd \& 3rd 'dredge-up' episodes (e.g., Becker \& Iben 1979,1980; Iben \& Renzini 1983). Ca, Si, Al, Mg and Fe have ratios to oxygen much lower than Solar, which is taken as due to depletion in grains.

The mean $\mathrm{O} / \mathrm{H}$ ratio for local $\mathrm{PNs}, 4.4 \times 10^{-4}$, agrees well with the value for H II regions (e.g., Orion) and M, S \& N-type red giants (Smith \& Lambert 1990a). However, the (well-determined) Solar photospheric value is a factor two higher. Are many $\mathrm{O}$ atoms in grains in nebulae, or is the nebular abundance scale wrong because large temperature fluctuations really exist? An interesting clue comes from analysis of B stars in the Orion cluster: Cunha \& Lambert (1992) found them to have the same $\mathrm{O} / \mathrm{H}$ ratio as the Orion nebula. It seems that the Sun may really be 'metal-rich' relative to the local ISM.

One issue still not resolved is whether, from patterns of CNO \& He, there is evidence that the $\mathrm{O}-\mathrm{N}$ part of the CNO cycle has operated. All papers disagree, some samples producing a finding that it has and others that it has not. The answer is that the evidence is still marginal and sample-dependent.

\section{The Galactic Bulge}

Webster (1988) analyzed 65 Bulge PNs, and found 'normal' abundances, and a ratio of Type I to other nebulae the same as in disk and Cloud samples. In a large sample of Bulge PNs, Ratag et al. (1992; and Ratag 1991) found most elements had abundances similar to local disk PNs, with marginal evidence for slightlyhigher $\mathrm{He} / \mathrm{H}$ and $\mathrm{N} / \mathrm{O}$ ratios. Walton et al. (Poster VI-237) also report 'disk-like abundances and a lack of $\mathrm{C}$-rich PNs in the Bulge. The most important result from these three studies is that the mean $\mathrm{O} / \mathrm{H}$ ratio in the Bulge samples is the same as for local disk PNs. This is suprising, since for K giants in the Bulge, Rich (1988) 
found a large spread of abundances - from 0.1 to $10 \times$ Solar - with a mean which was $2 \times$ Solar. Why is this not reflected in the PN oxygen value?

Two answers spring to mind. The chemical evolution of the Bulge could have been quite different from the local disk, with a different $\mathrm{O}-\mathrm{Fe}$ relation versus time being set up. However, in a recent model Matteucci \& Brocato (1990) predict that the effect will go the wrong way in galactic bulges, with $\mathrm{O}$ expected to be overabundant compared with Fe at about 'Solar' metallicity.

A second idea is that mentioned in the Introduction: that metal-rich stars tend not to make PNs. Ferguson \& Davidsen (1993) suggest that this could explain the $\mathrm{PN}-\mathrm{K}$ giant discrepancy in the Bulge: choosing a PN sample 'selects' the low end of any range in metallicity.

Because the discrepancy rests on only one stellar study, higher- dispersion spectroscopy of $\mathrm{K}$ giants is needed to confirm Rich's findings.

\section{The Galactic Halo}

Abundance patterns in these very-low abundance objects are important. The sample of extreme halo objects has been increased by four: IRAS1833-2357 (Gillett et al. 1989) in the globular cluster M22, PRMG-1 \& PRTM-1 (Peña et al. 1989, 1990), and M2-29 is a very low-abundance system (Webster 1988, Peña et al. 1991). Peimbert (1991a,b) discussed the abundances of He, C, N, O, Ne, S \& Ar in a sample of 10 such 'halo' PNs, including less extreme objects such as the metal-deficient NGC 4361 \& NGC 2242. IRAS1833 seems to be a 'born-again' object like A30, A58 \& A78, with highly-processed nebular material. M2-29 is a metal-poor object in the Bulge, presumably 'passing through' at high velocity.

As Peimbert shows, a few extreme halo objects show unusual abundance ratios. For example, although Henry (1990) showed the Ne/O ratio quite constant in PNs, the two glaring exceptions are the halo objects BB-1 (Ne/O high) and H4-1 ( Ne/O low). It is difficult to explain these ratios just through galactic chemical evolution effects, and I suggest that rather they reflect pollution by different individual massive stars in an inhomogeneous early halo.

\section{The Magellanic Clouds}

Reviews of Cloud PN ('MCPN') abundances are given by Dopita (1991; and these proceedings) and Barlow $(1989,1991)$. Fuller information is in Dopita's text.

Recent work includes three papers on optical line fluxes (Meatheringham \& Dopita 1991a,b; Vassiliadis et al. 1992) with the derived abundances and discussion given by Dopita \& Meatheringham (1991a,b). In this large programme the optical spectra of about 150 MCPN have now been measured. Torres-Peimbert et al. and de Freitas \& Costa (Posters VI-240 \& 242) report new abundance surveys of MCPNs. Kaler \& Jacoby $(1990,1991)$ compared the correlations between abundances and core mass in MCPN samples with dredge-up theory. Groenewegen \& de Jong (Poster VI-241) used parametrised, 'synthetic' AGB evolution models to predict MCPN abundance patterns - results from this useful work should be compared with the new observed abundance sets. 


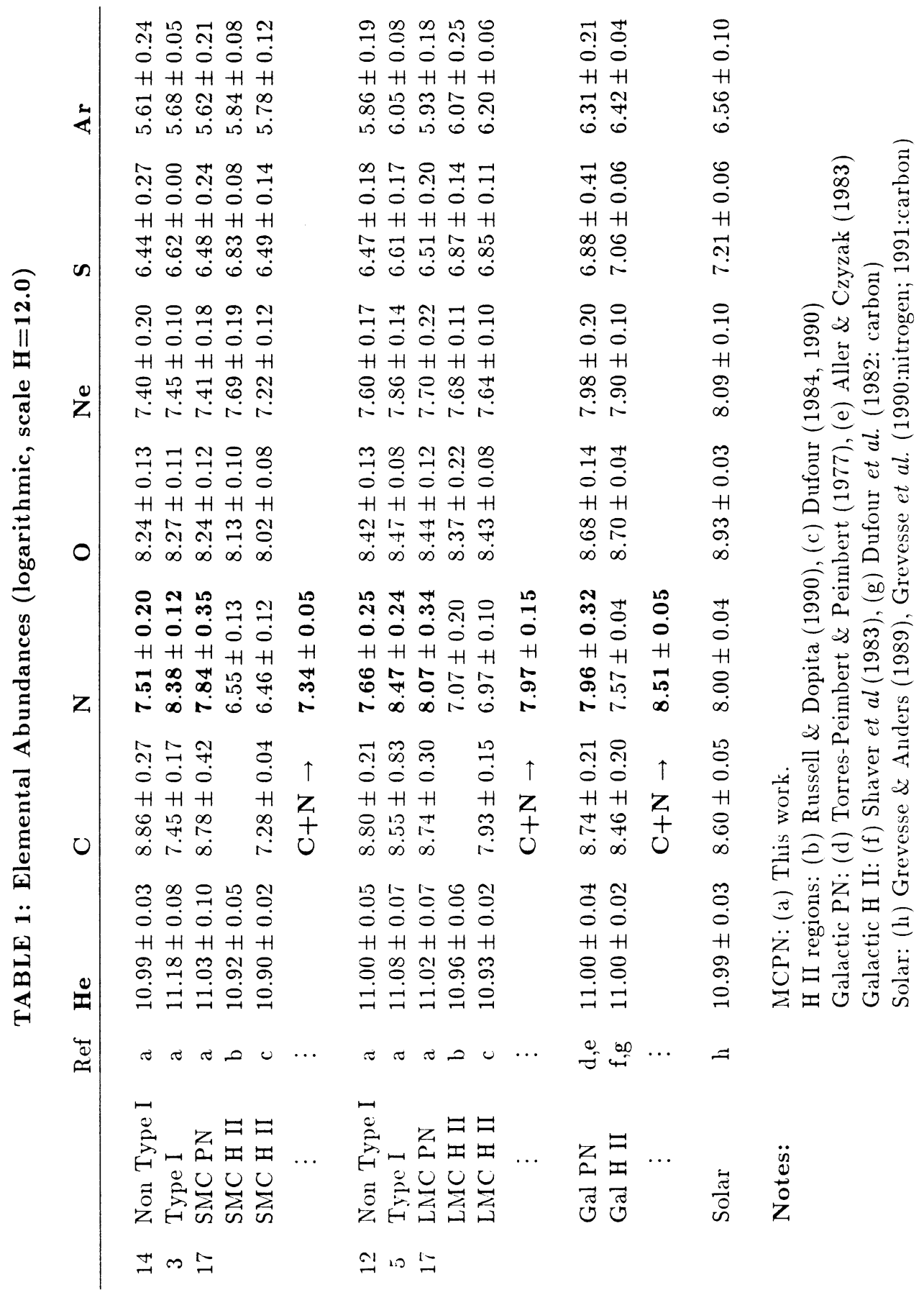


Walton, Barlow \& Clegg (1991, and in prep.) analysed 34 Cloud PN abundances, concentrating on $\mathrm{C} / \mathrm{H}$ ratios (which need IUE spectra). We update the work of Monk, Barlow \& Clegg (1988) with new AAT optical spectra and fresh, (plus all archived) IUE spectra. New ionization correction factors were derived from photoionization models of PNs with Cloud abundances.

Results are summarized in Table 1. Mean abundances for Type I and nonType I PNs in each Cloud, plus the Galactic disk, are compared with H II region abundances at the same galactic site. Detailed comparisons with results of Dopita and collaborators has not been made yet, so a summary of our results is given here.

As discussed by Barlow (1991) \& Clegg (1991), the mean CNO abundances can be interpreted simply by using the fact that the 1st and 2nd dredge-up episodes recycle the original $\mathrm{C}, \mathrm{N} \& \mathrm{O}$ in the stellar envelope. We take the initial composition of progenitor stars to be those of the relevant $\mathrm{H}$ II regions [this neglects any possible change through chemical evolution of the region, and also a current discrepancy between Cloud $\mathrm{C} / \mathrm{H}$ ratios for $\mathrm{H}$ II regions and stars, which disagree (Russell \& Dopita 1990, Pagel 1993)].

In the Table the sum of ' $\mathrm{C}+\mathrm{N}$ ' gives the available nitrogen abundance after $\mathrm{CN}$-cycle processing. Further $\mathrm{N}$ can only come from the $\mathrm{ON}$-cycle or from fresh ${ }^{12} \mathrm{C}$ created in the 3 rd dredge-up. However, since the mean $\mathrm{O} / \mathrm{H}$ ratios for all types of $\mathrm{PNs}$ and the $\mathrm{H}$ II regions in each system are the same within the errors, there is no evidence for ON-cycle processing (which would reduce $\mathrm{O}$ ). The conclusion is that the enhancement of $\mathrm{N}$ over the listed ' $\mathrm{C}+\mathrm{N}$ ' values is due to the latter effect, and thus that most of the Type I PNs suffered a 3rd dredge-up. Since many now have $\mathrm{C} / \mathrm{O}<1$ and high $\mathrm{N}$, their fresh ${ }^{12} \mathrm{C}$ was largely converted to ${ }^{14} \mathrm{~N}$.

Such analyses shows that the 'efficiency' of the both the 1 st \& 3rd dredge-ups increases at lower metallicities. 'Thus, while it is 'easier' to make a carbon star on the $\mathrm{AGB}$ at a given envelope mass when the $\mathrm{O} / \mathrm{H}$ ratio is low (a simple 'titration effect'), in fact this is added-to by the increased efficiency, which has also been noted in a number of theoretical studies (see article by Lattanzio).

Full details will be published. However, it should be noted that in our sample of 34 MCPNs, virtually all non-Type I PNs have $\mathrm{C} / \mathrm{O}>1$.

Attention is drawn to 3 SMC Type I PNs for which a 'standard analysis' yields very high values of $T_{e}\left(\mathrm{O}^{++}\right)(\sim 25,000 \mathrm{~K})$ and low abundances of all heavy elements (SMC N67, L305 \& L536; Walton et al.). Meatheringham et al. (1990) discovered this too for SMC L536 (=SMP 28), noting the apparently-low abundances, especially of carbon $\left(\log \mathrm{C}<6.0\right.$ !). However, the high $T_{e}\left(\mathrm{O}^{++}\right)$is hard to explain from photo-ionization alone, and moreover for the same nebulae $T_{e}\left(\mathrm{~N}^{+}\right)$is very much lower. Either these objects have high-density $\left(\geq 10^{5} \mathrm{~cm}^{-3}\right)$ central regions where the $\mathrm{O}^{++}$is located (with the $\mathrm{N}^{+}$further out), or some other process affects the [O III] lines. Perhaps, shock-excitation enhances the $4363 \AA$ line - one object (N67) is a strong X-ray source (Wang 1991). [The data of Table 1 are derived assuming these nebulae to have the average value of $T_{e}\left(\mathrm{O}^{++}\right)$from LMC Type I PNs].

It would be of great interest to increase the links between known types and PNs and AGB stars in the Clouds. Luminous AGB stars may provide one such link. Wood, Bessell \& Fox (1983) showed that at the bright end of the AGB ( $M_{B O L} \leq$ -6 ) in both Clouds, there were hardly any carbon stars and the (few) O-rich stars 
here commonly had MS or S characteristics. Smith \& Lambert (1990b) measured high lithium abundances in such AGB stars in the LMC \& SMC. The suggested interpretation was that they made Li during 'envelope burning'. This will also result in a hefty conversion of ${ }^{12} \mathrm{C}$ into ${ }^{14} \mathrm{~N}$ and ${ }^{13} \mathrm{C}$, and should result, after envelope ejection, in a Cloud Type I PN. The abundance patterns of these two sets of objects should be compared in more detail.

\section{Beyond the Magellanic Clouds}

Abundance analyses have been made for one nebula in the dwarf Fornax galaxy in the Local Group (Danziger et al. 1978, Maran et al. 1984) and of 3 PNs in M31 (Jacoby \& Ford 1986). The Fornax PN confirms the trend we noted earlier - in a metal-poor dwarf galaxy, it has a high carbon abundance $(\log \mathrm{O}=8.38, \log \mathrm{C}=8.95)$. Jacoby \& Ford (1986) analysed 3 PNs in M31, at projected distances of 3.5, 18 \& $33 \mathrm{kpc}$ from the centre. In $3-5 \mathrm{hr}$ exposures they were able to detect the important [O III $] 4363 \AA$ line and determine $\mathrm{T}_{e}$ values, but were unable to measure $\mathrm{N}_{e}$. Derived $\mathrm{O} / \mathrm{H}$ values were from $(1-5) \times 10^{-4}$, one nebula being a high-velocity (and thus halo-population) object, but still with disk-type $\mathrm{O}$ and $\mathrm{N}$ abundances.

Further advances in abundance studies within the Local Group (i.e., out to about $1 \mathrm{Mpc}$ ) will surely come from multi-object spectrographs (mainly, fibres and to a lesser extent, multi-slits). With $4 m$ telescopes and efficient, thinned CCD detectors, good progress can now be made. A greater challenge is to measure abundances in early-type galaxies beyond the Local group, say out to $3 \mathrm{Mpc}$ distance. Very long exposures with fibres and multi-slits may just be able to obtain a useful $\mathrm{S} / \mathrm{N}$ ratio for the crucial diagnostic lines of [O III] 4363 or [N II] $6584 \AA$.

In conclusion, studies of PN abundances as a function of site and metallicity have yielded some very important information; future advances can be expected mainly from Galactic Bulge, Magellanic Clouds, and other Local group galaxy samples. Observational tests of the possible lack of metal-rich PNs (and AGB stars) need to be developed.

\section{References}

Aller, L.H. \& Czyzak, S.J..: 1983, Ap. J. Suppl., 51, 211

Aller, L.H. \& Keyes, C.D.: 1987, Ap. J. Suppl., 65, 405

Barlow, M.J.: 1991, IAU Symp 148, eds. R. Haynes \& D.K. Milne, p291

Becker, S.A. \& Iben, I.: 1979, Ap. J., 232, 831

Becker, S.A. \& Iben, I.: 1980, Ap. J., 237, 111

Brocato, E., Matteucci, F., Mazzitelli, I. \& Tornambe, A.: 1990, Ap. J, 349, 458

Castellani, M. \& Tornambe, A.: 1991, Ap. J, 381, 393

Castellani, M., Limongi, M. \& Tornambe, A.: 1992, Ap. J, 389, 227

Clegg, R.E.S.: 1987, MNRAS, 229, 31P

Clegg, R.E.S.: 1989, IAU Symp 131, ed. S. Torres-Peimbert, p139

Clegg, R.E.S.: 1991, IAU Symp 145, eds. G. Michaud \& A. Tutukov, p387

Cunha, \& Lambert, D.L.: 1992, Ap. J., 399, 586

Dopita, M.A.: 1991, IAU Symp 148, eds. R. Haynes \& D.K. Milne, p299

Dopita, M.A. \& Meatheringham, S.J.: 1991a, Ap. J., 367, 115

Dopita, M.A. \& Meatheringham, S.J.: 1991b, Ap. J., 377, 480

Dufour, R.J.: 1984, IAU Symp. 108, p353

Dufour, R.J., Shields, G.A. \& Talbot, R.J.: 1982, Ap. J., 252, 461 
Faundez-Abans, M \& Maciel, W.: 1988, Rev. Mex. Astr. Astrof., 16, 105

Ferguson, H.C. \& Davidsen, A.F.: 1993, Ap. J, in press

de Freitas Pacheco, J.A., Maciel, W.J. \& Costa, R.: 1992, Astr. Astroph.

de Freitas Pacheco, J.A., Maciel, W.J., Costa, R. \& Barbuy, B.: 1991, Astr. Astroph, 250, 159

Gillett, F.C., et al.: 1989, Ap. J., 338, 862

Greggio, L. \& Renzini, A.: 1989, Ap. J, 364, 35

Henry, R.B.C.: 1989, MNRAS, 241, 453

Henry, R.B.C.: 1990, Ap. J., 356, 229

Iben, I. \& Renzini, A.: 1983, Ann Rev Astr. Astrophys., 21, 272.

Jacoby, G.H., \& Ford, H.C.: 1986, Ap. J., 304, 490

Jorissen, A., Smith, V.V. \& Lambert, D.L.: 1992, Astr. Astroph., 261, 164

Kaler, J.B. \& Jacoby, G.H.: 1990, Ap. J., 362, 491

Kaler, J.B. \& Jacoby, G.H.: 1991, Ap. J., 382, 134

Kaler, J.B., Shaw, R. \& Kwitter, K.: 1990, Ap. J., 359, 392

Koeppen, J., Acker, A. \& Stenholm,: 1991, Astr. Astroph, 248, 197

Liu, X-W. \& Danziger, I.J.: 1993, MNRAS, in press

Maciel, W.J.: 1991, in Elements and the Cosmos, eds. M.G. Edmunds \& R.J. Terlevich (CUP)

Maciel, W, de Freitas Pacheco, J.A. \& Landaberry, S.J.C.: 1990, Rev. Mex. Astr. Astrof., 21, 517

Manchado, A. \& Pottasch, S.R., 1989, Astr. Astroph, 222, 219

Maran, S.P., Gull, T.R., Stecher, T.P., Aller, L.H. \& Keyes, C.D.: 1984, Ap. J., 280, 615

Matteucci, F. \& Brocato, E.: 1990, Ap. J., 365, 539

Meatheringham, S.J. \& Dopita, M.A.: 1991a, Ap. J. Supp., 75, 407

Meatheringham, S.J. \& Dopita, M.A.: 1991b, Ap. J. Supp., 76, 1085

Meatheringham, S.J., et al.: 1990, Ap. J, 361, 101

Middlemass, D., Clegg, R.E.S., Walsh, J.R. \& Harrington, J.P.: 1991, MNRAS, 251, 284

Monk, D.J., Barlow, M.J. \& Clegg, R.E.S..: 1988, MNRAS, 234, 583

Peimbert, M.: 1990, Rep. Prog. Phys., 53, 1559

Peimbert, M.: 1991a, in Elements and the Cosmos, eds. M.G. Edmunds \& R.J. Terlevich (CUP)

Peimbert, M.: 1991b, in Highlights in Astronomy, Vol 9, (ed. J. Bergeron), (Dordrecht: Kluwer)

Peimbert, M. \& Torres-Peimbert, S.: 1987a, Rev. Mex. Astr. Astrof., 14, 540

Peimbert, M. \& Torres-Peimbert, S.: 1987b, Rev. Mex. Astr. Astrof., 15, 117

Peimbert, M., Sarmiento, A. \& Fierro, J.: 1991, PASP, 103, 815

Peña, M., Ruiz, M.T., Maza, J. \& Gonzalez, L.E.: 1989, Rev. Mex. Astr. Astrof., 17,

Peña, M., Torres-Peimbert, S. \& Ruiz,:1991, Rev. Mex. Astr. Astrof.,

Peña, M., Torres-Peimbert, S. \& Ruiz, M.T.: 1991, PASP, 1, 865

Perinotto, M.: 1992, Ap. J. Suppl., 76, 687

Ratag, M.A.: 1991, Ph.D. Thesis, University of Groningen

Ratag, M.A., Pottasch, S.R., Dennefeld, M. \& Menzies, J.: 1992, Astr. Astroph, 255, 255

Renzini, A. \& Voli, M.: 1981, Astr. Astroph, 94, 175

Rich, R.M.: 1998, A. J., 95, 828

Russell, S.C. \& Dopita, M.A.: 1990, Ap. J. Supp., 74, 93

Russell, S.C. \& Dopita, M.A.: 1992, Ap. J., 384, 508

Shaver, P.A., McGee, R.X., Danks, A.C. \& Pottasch, S.R.: 1983, MNRAS, 204, 53

Smith, V.V. \& Lambert, D.L.: 1990a, Ap. J. Supp., 72, 387

Smith, V.V. \& Lambert, D.L.: 1990b, Ap. J., 361, L69

Torres-Peimbert, S. \& Peimbert, M.: 1991, Rev. Mex. Astr. Astrof., 2, 181

Torres-Peimbert, S., Peimbert, M. \& Peña, M.:1991, Rev. Mex. Astr. Astrof., 15, 117

Torres-Peimbert, S., Peimbert, M. \& Peña, M.:1991, Astr. Astroph., 233, 540

Vassidialis, E., Dopita, M.A., Morgan, D.H. \& Bell, J.F.: 1992, Ap. J. Supp., 83, 87

Walton, N.A., Barlow, M.J. \& Clegg, R.E.S.: 1991, IAU Symp 148, eds. R. Haynes \& D. Milne, 291

Wang, Q.: 1991, MNRAS, 252, 47p

Webster, B.L.: 1988, MNRAS, 230, 377

Wood, P.R.: 1981, Physical Processes in Red Giants, ed. A. Renzini, p205

Zuckerman, B. \& Aller, L.H.: 1987, Ap. J., 301, 772 